\title{
OPTIMAL TRANSMISSION OF GAUSSIAN SIGNALS INVOLVING COST OF FEEDBACK
}

\author{
O.A. Glonti and L.G. Jamburia \\ Tbilisi I. Javakhishvili State University, Laboratory of Probabilistic-Statistical Methods \\ David Agmashenebeli Avenue 150a, 380012 Tbilisi, Georgia
}

Methods to analyse the problem of signal transmission through a noiseless feedback channel using the results of nonlinear filtering theory are given in [2]. In [1] we constructed an optimal transmission model for Gaussian signals taking into account the cost of transmission. The aim of this paper is to construct an optimal transmission scheme for a Gaussian signal involving the cost of feedback.

Suppose the transmitted message is a Gaussian random variable $\theta$ with $\mathbf{E} \theta=$ $m$ and $\mathbf{E}(\theta-m)^{2}=\gamma>0$, and the transmission of $\theta$ is carried out according to the following scheme

$$
\begin{gathered}
d \xi_{t}=\left\{\beta_{t}\left[A_{0}(t, \xi)+A_{1}(t, \xi) \theta\right]+\left(1-\beta_{t}\right)\left[B_{0}(t)+B_{1}(t) \theta\right]\right\} d t+d W_{t}, \\
\xi_{0}=0, \quad t \in[0, T]
\end{gathered}
$$

where $\beta=\left(\beta_{t}\right)_{t \leq T}$ is a $\left(\mathscr{F}_{t}^{\xi}\right)_{t \leq T}$-adapted stochastic process taking two values 0 and 1 . Under $\hat{\beta}_{t}=1$ the transmission is with feedback and under $\beta_{t}=0$ it is without feedback. Let

$$
\delta(t)=\inf _{A_{0}, A_{1}, B_{0}, B_{1}, \beta, \hat{\theta}} \mathbf{E}\left[\left(\theta-\hat{\theta}_{t}\right)^{2}+c \int_{0}^{t} \beta_{s} d s\right], \quad c>0,
$$

where $c$ is the cost of the feedback.

Let the following moment conditions

$$
\mathbf{E}\left\{\left[A_{0}(t, \xi)+A_{1}(t, \xi) \theta\right]^{2} \mid \mathscr{F}_{t}^{\xi}\right\} \leq P, \quad \mathbf{E}\left[B_{0}(t)+B_{1}(t) \theta\right]^{2} \leq P
$$

be satisfied, $P>0$.

The functionals $A_{0}, A_{1}, B_{0}, B_{1}, \beta$ are supposed to be such that equation (1) has a unique strong solution.

Denote

$$
m_{t}=\mathbf{E}\left(\theta \mid \mathscr{F}_{t}^{\xi}\right), \quad \gamma_{t}=\mathbf{E}\left[\left(\theta-m_{t}\right)^{2} \mid \mathscr{F}_{t}^{\xi}\right] .
$$


The equations for $m_{t}$ and $\gamma_{t}$ are of the following form

$$
\begin{aligned}
d m_{t}= & \gamma_{t}\left[\beta_{t} A_{1}(t, \xi)+\left(1-\beta_{t}\right) B_{1}(t)\right] \\
& \cdot\left\{d \xi_{t}-\left[\beta_{t} A_{0}(t, \xi)+\left(1-\beta_{t}\right) B_{0}(t)+\left(\beta_{t} A_{1}(t, \xi)\right.\right.\right. \\
& \left.\left.\left.+\left(1-\beta_{t}\right) B_{1}(t)\right) m_{t}\right] d t\right\}, \quad m_{0}=m
\end{aligned}
$$

$$
d \gamma_{t}=-\gamma_{t}^{2}\left[\beta_{t} A_{1}(t, \xi)+\left(1-\beta_{t}\right) B_{1}(t)\right]^{2} d t, \quad \gamma_{0}=\gamma
$$

Theorem. Suppose the transmission of a Gaussian variable $\theta$ is carried out according to scheme (1).

If $c \geq \frac{1}{4} \gamma P$, then the optimal strategy is $\beta_{s}^{*}=0, s \in[0, t]$, i.e., transmission without feedback. The optimal coding is $B_{1}^{*}(s)=\sqrt{P / \gamma}, B_{0}^{*}(s)=-m \sqrt{P / \gamma}$, and optimal decoding $m_{t}^{*}$, for given $\beta^{*}, B_{0}^{*}, B_{1}^{*}$, is determined through $(2)$ and (3). In that case

$$
\delta(t)=\frac{\gamma}{1+P t}
$$

Exactly the same statement is true if $c<\frac{1}{4} \gamma P$ and $t \leq a$ or $t \geq b$ with

$$
a=\frac{\gamma P-2 c-\sqrt{\gamma^{2} P^{2}-4 c \gamma P}}{2 c P}, \quad b=\frac{\gamma P-2 c+\sqrt{\gamma^{2} P^{2}-4 c \gamma P}}{2 c P} .
$$

If $c<\frac{1}{4} \gamma P$ and $t \in(a, b)$, then the optimal strategy has the form $\beta_{s}^{*}=$ $I\left(t-x_{0} \leq s \leq t\right)$, where $x_{0}$ is the unique solution of the equation

$$
c e^{P x}[1+P(t-x)]^{2}=\gamma P^{2}(t-x)
$$

in the interval $(0, t)$.

The optimal coding rules are determined as follows:

$$
\begin{gathered}
B_{1}^{*}(s)=\sqrt{\frac{P}{\gamma}}, \quad B_{0}^{*}(s)=-m \sqrt{\frac{P}{\gamma}} \\
A_{1}^{*}(s, \xi)=\sqrt{\frac{P}{\gamma}} \exp \left\{\frac{P}{2} \int_{0}^{s} \beta_{u}^{*} d u\right\}\left[1+\gamma \int_{0}^{s}\left(1-\beta_{u}^{*}\right)\left(B_{1}^{*}(u)\right)^{2} \exp \left\{-P \int_{0}^{u} \beta_{r}^{*} d r\right\} d u\right], \\
A_{0}^{*}(t, \xi)=-A_{1}^{*}(t, \xi) m_{t}^{*},
\end{gathered}
$$

where $m_{t}^{*}$ and $\gamma_{t}^{*}$ are determined through equations (3) and (4). In this case

$$
\delta(t)=\frac{\gamma e^{-P x_{0}}}{1+P\left(t-x_{0}\right)}+c x_{0} .
$$

To prove this theorem we need the following lemma. 
Lemma. Let $\beta=\left(\beta_{t}\right)_{t \geq 0}$ be a real function with values in $[0,1]$ and let $P$ be a positive constant. Then the following inequality is valid

$$
\int_{0}^{t} e^{-P \int_{0}^{s} \beta_{u} d u} d s \leq t-\int_{0}^{t} \beta_{s} d s+\frac{1}{P}\left(1-e^{-P \int_{0}^{t} \beta_{s} d s}\right) .
$$

If $\beta$ has the form $\beta_{s}=I(u \leq s \leq t), u \in[0, t]$, then the inequality reduces into an equality.

Proof. Let $\int_{0}^{t} \beta_{s} d s=v$ and $\beta_{s}^{*}=I(t-v \leq s \leq t)$. Then $\int_{0}^{t} \beta_{s}^{*} d s=v$. Let us first show that

$$
\int_{0}^{s} \beta_{u}^{*} d u \leq \int_{0}^{s} \beta_{u} d u
$$

for any $s \in[0,1]$. This inequality is evident for $s<t-v$. Assume that for some $t_{1} \geq t-v$ an inverse inequality occurs. Then

$$
v=\int_{0}^{t} \beta_{u}^{*} d u=\int_{0}^{t_{1}} \beta_{u}^{*} d u+\int_{t_{1}}^{t} \beta_{u}^{*} d u>\int_{0}^{t_{1}} \beta_{u} d u+\int_{t_{1}}^{t} \beta_{u} d u=\int_{0}^{t} \beta_{u} d u
$$

which is not true. Thus,

$$
\int_{0}^{t} e^{-P \int_{0}^{s} \beta_{u} d u} d s \leq \int_{0}^{t} e^{-P \int_{0}^{s} \beta_{u}^{*} d u} d s
$$

One can easily see that

$$
\int_{0}^{t} e^{-P \int_{0}^{s} \beta_{u}^{*} d u} d s=t-v+\frac{1}{P}\left(1-e^{-P v}\right)
$$

and since $v=\int_{0}^{t} \beta_{s} d s$, we obtain the required inequality.

The validity of the second assertion of the lemma follows by a direct verification.

Proof of the theorem. For fixed coding functionals $A_{0}, A_{1}, B_{0}, B_{1}$ and strategy $\beta$ the optimal decoding is $m_{t}$. Hence,

$$
\delta(t)=\inf _{A_{0}, A_{1}, B_{0}, B_{1}, \beta} \mathbf{E}\left[\gamma_{t}+c \int_{0}^{t} \beta_{s} d s\right] .
$$

We construct first the optimal coding functionals for a fixed strategy $\beta$. Rewrite the moment condition in the following form

$$
\left[A_{0}(t, \xi)+A_{1}(t, \xi) m_{t}\right]^{2}+\gamma_{t} A_{1}^{2}(t, \xi) \leq P, \quad\left[B_{0}(t)+B_{1}(t) m\right]^{2}+\gamma B_{1}^{2}(t) \leq P .
$$


The equation for $\gamma_{t}$ can be written in the form

$$
\gamma_{t}=\gamma \exp \left\{-\int_{0}^{t} \beta_{s} \gamma_{s} A_{1}^{2}(s, \xi) d s-\int_{0}^{t}\left(1-\beta_{s}\right) \gamma_{s} B_{1}^{2}(s) d s\right\} .
$$

From (5) it follows that $\gamma_{t} A_{1}^{2}(t, \xi) \leq P$. Therefore,

$$
\gamma_{t} \geq \gamma \exp \left\{-P \int_{0}^{t} \beta_{s} d s-\int_{0}^{t}\left(1-\beta_{s}\right) B_{1}^{2}(s) d s\right\} .
$$

If we choose $A_{1}^{*}(t, \xi)=\sqrt{P / \gamma_{t}^{*}}$, where $\gamma_{t}^{*}$ is a solution of (4) for a given functional $A_{1}^{*}$ and a fixed $B_{1}$, then the inequality reduces into an equality. Hence $A_{1}^{*}$ is the optimal coding. The moment condition is satisfied for $A_{0}^{*}(t, \xi)=-A_{1}^{*}(t, \xi) m_{t}^{*}$, where $m_{t}^{*}$ is a solution of (3) for given $A_{0}^{*}, A_{1}^{*}, \gamma_{t}^{*}$. If we solve the equation (4) for $A_{1}^{*}(t, \xi)=\sqrt{P / \gamma_{t}^{*}}$, we obtain

$$
\gamma_{t}^{*}=\frac{\gamma e^{-P \int_{0}^{t} \beta_{s} d s}}{1+\gamma \int_{0}^{t}\left(1-\beta_{s}\right) B_{1}^{2}(s) e^{-P \int_{0}^{s} \beta_{u} d u} d s},
$$

and therefore the optimal coding has the form

$$
A_{1}^{*}(t, \xi)=\sqrt{\frac{P}{\gamma}} e^{P / 2 \int_{0}^{t} \beta_{s} d s}\left[1+\gamma \int_{0}^{t}\left(1-\beta_{s}\right) B_{1}^{2}(s) e^{-P \int_{0}^{s} \beta_{u} d u} d s\right] .
$$

Thus

$$
\delta(t)=\inf _{B_{0}, B_{1}, \beta} \mathbf{E}\left[\frac{\gamma e^{-P \int_{0}^{t} \beta_{s} d s}}{1+\gamma \int_{0}^{t}\left(1-\beta_{s}\right) B_{1}^{2}(s) e^{-P \int_{0}^{s} \beta_{u} d u} d s}+c \int_{0}^{t} \beta_{s} d s\right] .
$$

If follows from (5) that $B_{1}^{2}(t) \leq P / \gamma$. If we choose $B_{1}^{*}(t)=\sqrt{P / \gamma}$ and $B_{0}^{*}(t)=$ $-m \sqrt{P / \gamma}$, then the condition (5) is fulfilled and

$$
\delta(t)=\inf _{\beta} \mathbf{E}\left[\frac{\gamma e^{-P \int_{0}^{t} \beta_{s} d s}}{1+P \int_{0}^{t}\left(1-\beta_{s}\right) e^{-P \int_{0}^{s} \beta_{u} d u} d s}+c \int_{0}^{t} \beta_{s} d s\right] .
$$

Thus, for a fixed strategy $\beta$, our construction gives the optimal coding functionals and the optimal decoding.

Now we construct the optimal strategy. It can be easily shown that

$$
\delta(t)=\inf _{\beta} \mathbf{E}\left[\frac{\gamma}{1+P e^{P \int_{0}^{t} \beta_{s} d s} \int_{0}^{t} e^{-P \int_{0}^{s} \beta_{u} d u} d s}+c \int_{0}^{t} \beta_{s} d s\right] .
$$


We denote the expression in the brackets by $\delta_{\beta}(t)$. Using the inequality from the previous lemma we obtain

$$
\delta_{\beta}(t) \geq \frac{\gamma e^{-P \int_{0}^{t} \beta_{s} d s}}{1+P\left(t-\int_{0}^{t} \beta_{s} d s\right)}+c \int_{0}^{t} \beta_{s} d s .
$$

Consider the function

$$
f(x)=\frac{\gamma e^{-P x}}{1+P(t-x)}+c x, \quad x \in[0, t] .
$$

The properties of the derivatives of this function easily show that this function increases for all $x \in[0,1]$, if $c \geq \frac{1}{4} \gamma P$ and therefore $f(x) \geq f(0)=\gamma /(1+P t)$. If $c<\gamma P / 4$ and $t \notin(a, b)$, where the numbers $a$ and $b$ have been defined in the enunciation of the theorem, then again $f(x) \geq \gamma /(1+P t), x \in[0, t]$. If $c<\frac{1}{4} \gamma P$ and $t \in(a, b)$, then $f^{\prime}(x)<0$ for $x \in\left[0, x_{0}\right)$ and $f^{\prime}(x)>0$ for $x \in\left(x_{0}, t\right]$, i.e., $f(x) \geq f\left(x_{0}\right), x \in[0, t]$, where $x_{0}$ is the unique solution of the equation

$$
c e^{P x}[1+P(t-x)]^{2}=\gamma P^{2}(t-x) .
$$

Using the properties of the function $f$ we can obtain the following statements: If $c \geq \frac{1}{4} \gamma P$, then for any strategy $\beta$ we have

$$
\delta_{\beta}(t) \geq \frac{\gamma}{1+P t}, \quad\left(\text { note that } 0 \leq \int_{0}^{t} \beta_{s} d s \leq t\right),
$$

and for $\beta_{s}^{*}=0, s \in[0, t]$,

$$
\delta_{\beta^{*}}(t)=\frac{\gamma}{1+P t},
$$

i.e., $\beta^{*}$ is the optimal strategy.

If $c<\frac{1}{4} \gamma P$ and $t \leq a$ or $t \geq b$, then the same results hold.

If $c<\frac{1}{4} \gamma P$ and $t \in(a, b)$, then

$$
\delta_{\beta}(t) \geq \frac{\gamma e^{P x_{0}}}{1+P\left(t-x_{0}\right)}+c x_{0} .
$$

Let $\beta_{s}^{*}=I\left(t-x_{0} \leq s \leq t\right)$. Then, according to the second statement in the previous lemma, we obtain

$$
\begin{aligned}
\delta_{\beta^{*}}(t) & =\frac{\gamma e^{-P \int_{0}^{t} \beta_{s}^{*} d s}}{1+P\left(t-\int_{0}^{t} \beta_{s}^{*} d s\right)}+c \int_{0}^{t} \beta_{s}^{*} d s \\
& =\frac{\gamma e^{-P x_{0}}}{1+P\left(t-x_{0}\right)}+c x_{0},
\end{aligned}
$$

which completes the proof of the theorem. 


\section{References}

[1] Glonti, O.A., and L.G. Jamburia: On the optimal transmission of signals involving the cost for transmission. - Fifth International Vilnius Conference on Probability Theory and Mathematical Statistics, Abstracts of Communications III, Vilnius, 1989, 142143 (Russian).

[2] Liptser, R.Sh., and A.N. Shiryaev: Statistics of random processes I-II. - SpringerVerlag, New York, 1977/1978 (English translation). 